\title{
Ghost spintronic THz-emitter-array microscope
}

\author{
Si-Chao Chen ${ }^{1,2}$, Zheng Feng ${ }^{3}$, Jiang $\mathrm{Li}^{1,3}$, Wei Tan ${ }^{3}$, Liang-Hui Du ${ }^{1,3}$, Jianwang Cai ${ }^{4}$, Yuncan Ma ${ }^{1}$, Kang $\mathrm{He}^{5}$, \\ Haifeng Ding $\mathbb{1}^{5}$, Zhao-Hui Zhai ${ }^{1,3}$, Ze-Ren Li ${ }^{1}$, Cheng-Wei Qiu' ${ }^{6}$ Xi-Cheng Zhang $\mathbb{1}^{7}$ and Li-Guo Zhu ${ }^{1,3}$
}

\begin{abstract}
Terahertz $(\mathrm{THz})$ waves show great potential in nondestructive testing, biodetection and cancer imaging. Despite recent progress in $\mathrm{THz}$ wave near-field probes/apertures enabling raster scanning of an object's surface, an efficient, nonscanning, noninvasive, deep subdiffraction imaging technique remains challenging. Here, we demonstrate $\mathrm{THz}$ near-field microscopy using a reconfigurable spintronic THz emitter array (STEA) based on the computational ghost imaging principle. By illuminating an object with the reconfigurable STEA followed by computing the correlation, we can reconstruct an image of the object with deep subdiffraction resolution. By applying an external magnetic field, inline polarization rotation of the THz wave is realized, making the fused image contrast polarization-free. Time-of-flight (TOF) measurements of coherent $\mathrm{THz}$ pulses further enable objects at different distances or depths to be resolved. The demonstrated ghost spintronic THz-emitter-array microscope (GHOSTEAM) is a radically novel imaging tool for $\mathrm{THz}$ near-field imaging, opening paradigm-shifting opportunities for nonintrusive label-free bioimaging in a broadband frequency range from 0.1 to $30 \mathrm{THz}$ (namely, $3.3-1000 \mathrm{~cm}^{-1}$ ).
\end{abstract}

\section{Introduction}

The unique properties of terahertz waves $(0.1-10 \mathrm{THz})^{1,2}$, such as the nonionizing photon energy, spectral fingerprint, and transparency for most nonpolar materials, have attracted much research interest and enabled many applications, such as nondestructive testing ${ }^{3}$, biodetection ${ }^{4-6}$, and cancer imaging ${ }^{7}$. However, the long wavelength of $\mathrm{THz}$ waves $(1 \mathrm{THz} \sim 300 \mu \mathrm{m})$ typically limits the resultant imaging resolution to greater than the millimetre scale in conventional far-field imaging methods due to the wellknown Rayleigh diffraction limit, therefore restricting their use in many emergent applications such as cellular imaging. On the other hand, near-field imaging by mapping the object-modulated evanescent waves paves the way towards deep subwavelength resolution and is particularly desirable at long wavelengths, for example, at THz frequencies. AFM-

Correspondence: Li-Guo Zhu (zhuliguo@tsinghua.org.cn)

${ }^{1}$ Institute of Fluid Physics, China Academy of Engineering Physics, Mianyang 621900 Sichuan, China

${ }^{2}$ Department of Optics and Optical Engineering, University of Science and Technology of China, Hefei 230026 Anhui, China

Full list of author information is available at the end of the article

These authors contributed equally: Si-Chao Chen, Zheng Feng, Jiang Li,

Wei Tan or STM-tip-enhanced $\mathrm{THz}$ probes ${ }^{8,9}$ or microantenna/ aperture $\mathrm{THz}$ probes ${ }^{10}$ have achieved micrometre- or even atomic-scale resolution ${ }^{9}$. Nevertheless, these techniques require mechanical raster scanning of the surface of an object pixel by pixel, with a relatively poor signal-to-noise ratio (SNR). Recently, near-field ghost imaging techniques $^{11-14}$ have been experimentally demonstrated to increase the SNR by $\sqrt{N}$ times (where $N$ denotes the pixel number of a digital picture) over that in raster scanning ${ }^{15}$. In these approaches, $\mathrm{THz}$ images are spatially encoded in the near field with deterministic patterns (e.g., the WalshHadamard matrix ${ }^{11-17}$ ). Then, the total intensities (or electric field amplitudes) of encoded $\mathrm{THz}$ images are collected and detected with a single-pixel detector in the far field. After postprocessing via computational algorithms to correlate the detected $\mathrm{THz}$ intensities (or electric field amplitudes) with the deterministic patterns ${ }^{18,19}$, near-field images can be reconstructed. In this scheme, the subwavelength spatial information "hidden" in the diffracted far-field distribution can be recovered from the intensities (or amplitude fields) recorded by a mere single-pixel detector. 
To encode $\mathrm{THz}$ images in a single pixel, the conventional method is to use photogenerated spatial $\mathrm{THz}$ wave modulators as reconfigurable masks ${ }^{11-13}$. However, the $\mathrm{THz}$ wave amplitude passing through the subwavelength apertures of modulators follows the scaling rule $1 / a^{3}$ (where $a$ denotes the diameter of the apertures on the mask ${ }^{20}$, which fails to image deep subwavelength structures.

Alternatively, directly detecting $\mathrm{THz}$ images ${ }^{14}$ or generating patterned THz waves ${ }^{16,17}$ by encoding femtosecond (fs) laser pulses in nonlinear electrooptic (EO) crystals (such as ZnTe) has been proposed to bypass the scaling rule. However, subwavelength structures are rapidly blurred upon propagation in hundreds of micrometre-thick EO crystals ${ }^{14}$ (Supplementary Fig. S1). Recently, the time-resolved single-pixel detection of $\mathrm{THz}$ pulses has been theoretically proposed ${ }^{16}$ and experimentally demonstrated ${ }^{17}$ to recover higher-resolution images. However, suffering from the milimeter-scale-thick nonlinear crystal, THz-wave generation along with diffraction occurs across the entire volume of the crystal, leading to a limited spatial resolution of 50-100 $\mu \mathrm{m}$. Although the spatial resolution can be further improved by the inverse propagation algorithm, this requires full-wave measurements of $\mathrm{THz}$ pulses in the time domain with extra cost in terms of detection resources and involves an inverse problem. Furthermore, it only applies to recovering 2D-structured images. In addition, constrained by the electromagnetic boundary conditions (Supplementary Fig. S2), the distribution of the polarized THz field in subwavelength structures presents a "distorted" image $^{11-14}$ that might lead to misrecognition of the object's morphology.

In this work, we utilize a spintronic THz emitter (STE) to illuminate an object in the near field. STEs are a novel type of $\mathrm{THz}$ emitter based on the spin-related effects ${ }^{21,22}$ in ferromagnetic/nonmagnetic (FM/NM) heterostructures ${ }^{23-26}$, which are only a few nanometres thick but offer generation efficiency comparable to conventional milimetre-thick EO crystals. In principle, an STE can fully cover the $0.1-30 \mathrm{THz}$ frequency range ${ }^{24}$ without phonon absorption, which is superior to all the current solid emitters. Limited by the 1mm-thick ZnTe used as the detector $(3-\mathrm{THz}$ detection bandwidth) and the pulse duration of the pump laser ( $90 \mathrm{fs})$, the STE in our work provided a bandwidth of up to $2 \mathrm{THz}$. To date, all applications of STEs have only focused on their farfield properties, whereas the highly efficient few-nanometrethick STE is capable of illuminating an object at an extremely near field, which naturally breaks the diffraction limit. The key challenge for using STEs in near-field imaging is how to map the object-modulated $\mathrm{THz}$ field without near-field scanning probes. In view of this, we developed a near-field illuminating "array" (STEA) whose "elements" are coherent and individually programmable in binary states of either "on" or "off" by photoexcitation. Combining the programmable near-field illuminating STEA and far-field single-pixel detection, we designed and demonstrated a novel ghost spintronic $\mathrm{THz}-$ emitter-array microscope (GHOSTEAM) for $\mathrm{THz}$ wave imaging with deep subdiffraction resolution. A minimum resolution of $6.5 \mu \mathrm{m}$ at a single pixel was demonstrated with a contrast of more than $57 \pm 21 \%$ ( $>20 \%$ required by the Rayleigh criterion ${ }^{14}$ ) in a $6-\mu$ m metal gap. In addition, polarization effects on the subdiffraction-limited image were eliminated via postprocessing of two images with mutually orthogonal polarizations. In addition, TOF microscopic topography was demonstrated with a 3D silica structure.

\section{Results \\ Concept design}

In our design, as shown in Fig. 1a, the STEA under external magnetic field $\boldsymbol{B}$ generates spatially structured $\mathrm{THz}$ pulses upon fs-laser-pulse spatial photoexcitation. The STEA, consisting of a W( $(2 \mathrm{~nm}) / \mathrm{Fe}(2 \mathrm{~nm}) / \mathrm{Pt}(2 \mathrm{~nm})$ trilayer heterostructure on a transparent $\mathrm{MgO}$ substrate (Fig. 1b), provides great $\mathrm{THz}$ conversion efficiency in terms of output amplitude, comparable to that of $1-\mathrm{mm}$ $\mathrm{ZnTe}^{24}$. The STEA is capped with a $150-\mathrm{nm} \mathrm{SiO}_{2}$ layer $(n=1.97)$ to protect it from being damaged by the fs laser. The output $\mathrm{THz}$ electric field $\boldsymbol{E}(t)$ is linearly polarized perpendicular to the applied magnetic field $\boldsymbol{B}$, as described by $\boldsymbol{E}(t) \propto\left(\boldsymbol{J}_{c}=\boldsymbol{J}_{s} \times \boldsymbol{B}\right)^{21-26}$, where $\boldsymbol{J}_{s}$ represents the spin current induced by the fs laser and $J_{\mathrm{c}}$ represents the charge current converted in the NM metals under $\boldsymbol{B}$. To perform ghost imaging (see "Materials and methods" for details), the Walsh-Hadamard matrix ${ }^{27}$ was used to code the STEA due to its unrivalled noise suppression performance among various measurement matrices ${ }^{11-17}$. The patterns, programmed in the order of the Walsh-Hadamard masks, were spatially encoded on the excitation fs laser beam by a digital micromirror device (DMD) with a switching time of $5 \mu \mathrm{s}$. On the exit surface of the STEA, the profile of the output $\mathrm{THz}$ pulse is as accurate as that of the excitation fs laser because the $150 \mathrm{~nm}$ propagation distance in the $\mathrm{SiO}_{2}$ protective layer is too thin for the $\mathrm{THz}$ wave to be diffracted $(150 \mathrm{~nm} \approx$ $5 \times 10^{-4} \lambda_{0} / n$, where $\lambda_{0}=600 \mu \mathrm{m}$ and $n=1.97$; see Supplementary Fig. S1 for the theoretical calculation of the near-field evanescent wave). The spatial near-field profile of the THz pulse from the STEA consists of individually real-time programmable "elements" up to $128 \times 128$ (see Supplementary Fig. S3) in either "on" or "off" binary emission states, as shown in Fig. 1b. The pixel size of each "element" is $6.5 \mu \mathrm{m} \times 6.5 \mu \mathrm{m}$ in our experiments, which can be zoomed in or out by using an optical projection imaging system depending on the practical requirements. After being transmitted through an object placed in the near-field region $(z=150 \mathrm{~nm})$, the structured $\mathrm{THz}$ pulses were collected and focused onto a 1-mm-thick (110) ZnTe crystal for single-pixel coherent detection by EO 

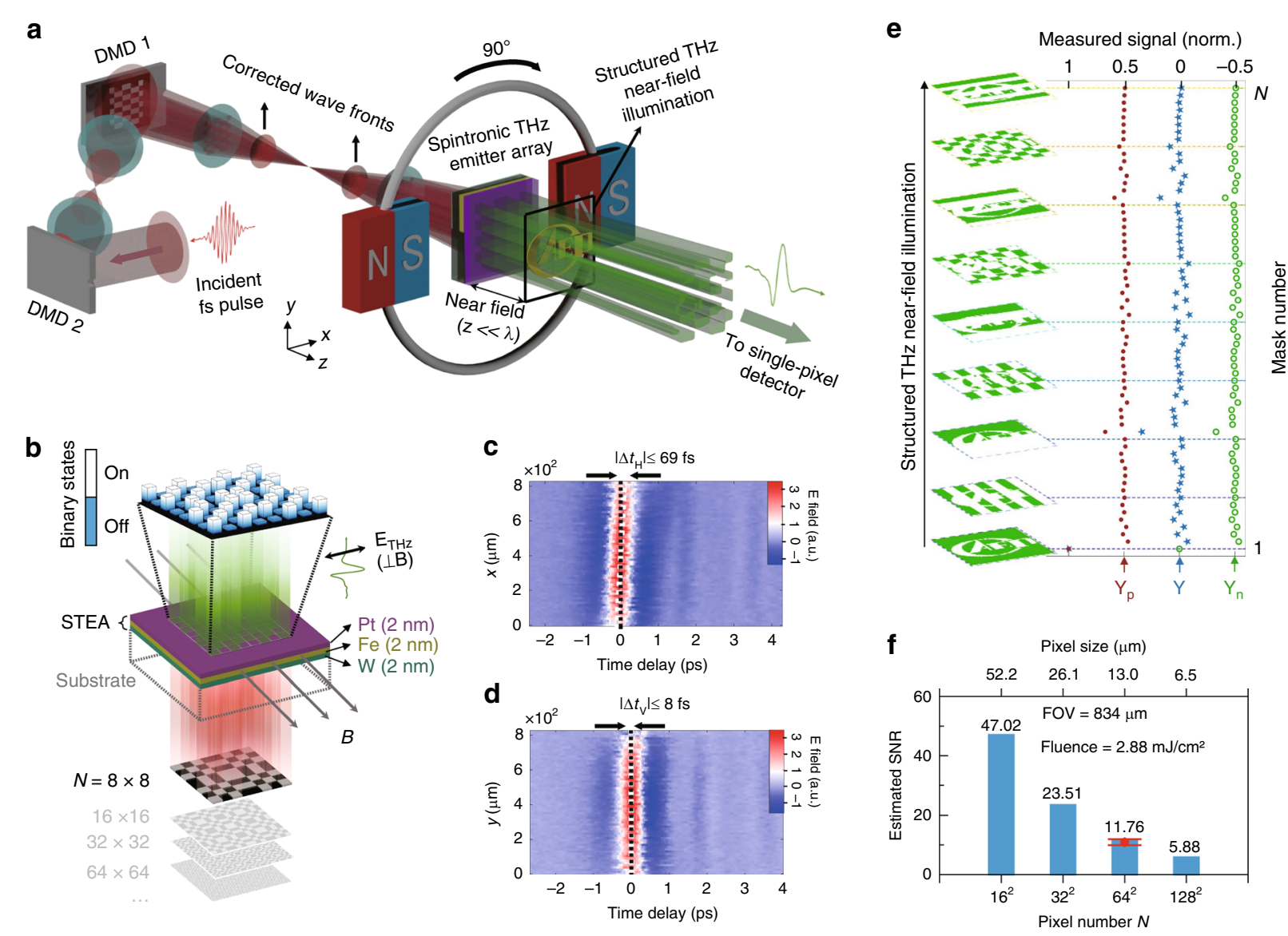

Fig. 1 Schematic of the GHOSTEAM system. a Schematic of the GHOSTEAM system. The spintronic THz emitter array (STEA) is excited by twoDMD-encoded $f s$ laser pulses and generates spatially coded THz pulses. An object "CAEP" was placed in the near-field region $(z \ll \lambda)$. The illuminating $\mathrm{THz}$ pulse was collected and sent to a single-pixel detector. $\mathbf{b}$ Schematic of the STEA, consisting of a W( $(2 \mathrm{~nm}) / \mathrm{Fe}(2 \mathrm{~nm}) / \mathrm{Pt}(2 \mathrm{~nm}) \operatorname{trilayer}$ heterostructure and working in the binary emission state with polarization perpendicular to applied magnetic field $\boldsymbol{B}$. Spatiotemporal THz waveform along the horizontal (c) and vertical (d) directions. The wavefront is indicated by white dotted lines, and time $t=0$ is indicated by black dotted lines. $\left|\Delta t_{H}\right|$ and $\left|\Delta t_{\mathrm{V}}\right|$ are the temporal delays in the horizontal and vertical directions, respectively. e Schematic of the detection for ghost imaging, and measured signal $\mathbf{Y}$ (which is the difference between the positive mask value and negative mask value, $\mathbf{Y}=\left|\mathbf{Y}_{\mathrm{p}}\right|-\left|\mathbf{Y}_{\mathrm{n}}\right|$, in the case of Hadamard multiplexing) from the single-pixel detector for an object "CAEP" illuminated by a sequence of prearranged structured THz waves. $\mathbf{f}$ Estimated SNRs as a function of pixel number $N$ under the condition of a pump fluence of $2.88 \mathrm{~mJ} \mathrm{~cm}{ }^{-2}, F_{O V}=834 \mu \mathrm{m} \times 834 \mu \mathrm{m}$. The red marker with a value of $10.92 \pm 0.97$ is the experimental result (see the main text below and Supplementary section 4 for details).

sampling. The peak electric amplitudes were recorded for reconstructing $\mathrm{THz}$ subdiffraction ghost images.

After reflection upon a DMD, the fs laser pulse suffers from wavefront tilt in the DMD deflection direction (horizontal) since the DMD is essentially a reflective blazed grating ${ }^{28}$. The resultant fs laser pulse with a tilted wavefront will badly lose its temporal coherence among individual micromirrors along the deflection direction. To correct the wavefront tilt, another $\mathrm{DMD}\left(\mathrm{DMD}_{2}\right)$ was imaged onto the encoding $\mathrm{DMD}\left(\mathrm{DMD}_{1}\right)$ by a $4 f$ system with a 1:1 imaging ratio, as shown in Fig. 1a. Due to the fixed time delay of ghost imaging (corresponding to the peak of the $\mathrm{THz}$ pulses), the DMD-induced temporal smearing should be minimized. Otherwise, decoherence (large time delay in the wavefront) would result in severely distorted images (see Supplementary Fig. S6). The temporal delay in the horizontal direction $\left|\Delta t_{\mathrm{H}}\right|$ was ultimately corrected to less than $69 \pm 13 \mathrm{fs}$ (Fig. 1c) but could not be completely eliminated due to the manufacturing tolerance of the tilt angles of the two DMDs (see Supplementary Fig. S6). Meanwhile, the temporal delay in the vertical direction $\left|\Delta t_{\mathrm{V}}\right|$ was measured to be less than $8 \mathrm{fs}$ (Fig. 1d), which is expected because the two DMDs only deflect in the horizontal direction. The spatiotemporal $\mathrm{THz}$ waveforms (Fig. 1c, d) were measured by ghost imaging using 64-order Walsh-Hadamard coding 1-D masks (see "Materials and methods" and Supplementary Fig. S5 for detailed information and Supplementary GIFs. 1 and 2 for raw data).

An object was illuminated by the coherent programmable STEA using a sequence of prearranged Walsh-Hadamard masks, and the computational ghost 
imaging algorithm was applied to correlate the peak amplitude measured by a single-pixel detector with the sequence of predetermined incident $\mathrm{THz}$ wave patterns (Fig. 1e, with the detailed procedure shown later). The reconfigurable area on the STEA, defined as the first imaging field of view $\mathrm{FOV}_{1}$, was measured as $834 \mu \mathrm{m} \times$ $834 \mu \mathrm{m}$, with up to $128 \times 128$ coding pixels. The SNRs of reconstructed images encoded by Walsh-Hadamard masks were numerically determined as a function of the pixel number (or pixel size in $\mathrm{FOV}_{1}$; see "Materials and methods"), which gave a reasonable value of $\sim 47.02$ when $\mathrm{FOV}_{1}$ was coded into $16 \times 16$ pixels under our experimental conditions (pump fluence $\sim 2.88 \mathrm{~mJ} \mathrm{~cm}^{-2}$, dynamic range of peak field $\sim 1043$, and pulse fluctuation $\sim 0.7 \%$ ), as shown in Fig. 1f. It is worth noting that although the detected $\mathrm{THz}$ band was less than $2 \mathrm{THz}$ due to the 90 -fs pulse and 3-THz detection bandwidth (1-mm $\mathrm{ZnTe}$ ), the STE has demonstrated complete coverage of the entire $\mathrm{THz}$ region, i.e., $0.1-30 \mathrm{THz}$, when a shorter pump pulse (10 fs) and a wide-band detector are used ${ }^{24,26}$. More experimental details are given in "Materials and methods" and Supplementary section 2.

\section{Subdiffraction ghost imaging}

The STEA was experimentally reconfigured in a $64 \times$ 64-order Walsh-Hadamard matrix sequence to acquire $\mathrm{THz}$ subdiffraction-limited images of an object (see Fig. 2a) positioned $z=150 \mathrm{~nm}$ from the STEA (thickness of the protective $\mathrm{SiO}_{2}$ layer on top of the STEA). The field amplitudes of spatially coded $\mathrm{THz}$ waves that passed through the object were measured at a fixed time delay of 0 ps (as indicated by the black dotted lines in Fig. 1c, d). The reconstructed $64 \times 64$ ghost images in $\mathrm{FOV}_{1}=$ $834 \mu \mathrm{m} \times 834 \mu \mathrm{m}$ with mutually orthogonal polarizations are shown in Fig. $2 \mathrm{~b}$, c, whose pixel sizes are both $13.0 \mu \mathrm{m} \times 13.0 \mu \mathrm{m}$. The reconstructed $64 \times 64$ ghost image in a smaller area $\mathrm{FOV}_{2}=417 \mu \mathrm{m} \times 417 \mu \mathrm{m}$ (indicated by the black dashed box in Fig. 2a) with a smaller pixel size of $6.5 \mu \mathrm{m} \times 6.5 \mu \mathrm{m}$ is shown in Fig. $2 \mathrm{~d}$. The $y$ dependent $\mathrm{THz}$ field distribution across the slit region (indicated by the black dashed arrow in Fig. 2b) was extracted from the associated pixels, as shown in Fig. 2e. Note that every pixel value was averaged from the identical-row pixels within the slit region, with prior knowledge about the object's horizontal homogeneity in the slit region. A contrast ratio of $57 \pm 21 \% \quad(>20 \%$ required by the Rayleigh criterion ${ }^{14}$ for distinguishing slits) was observed in the narrowest $6-\mu \mathrm{m}$ metal slit within $\mathrm{FOV}_{2}$ (see Supplementary section 5 for the quantification process and Supplementary GIFs. 3-5 for the raw data), which clearly proves that our GHOSTEAM system deeply breaks the diffraction barrier of $\sim 366 \mu \mathrm{m}$ for the $600-\mu \mathrm{m}$ $\mathrm{THz}$ wave (Rayleigh resolution of $0.61 \lambda_{0} / \mathrm{NA}$ ). The spatial resolution of the GHOSTEAM system using this experimental setup is limited to the minimum available pixel size of $6.5 \mu \mathrm{m}$, which depends on the accuracy of the mask patterns on the STEA projected by the DMD. As the micromirrors of the DMD are arranged in the diamond orientation, the accuracy of the coding profile on the STEA is decreased since a mask pixel is formed by fewer micromirrors (see Supplementary Fig. S3). Regardless of the diffraction of the coding optical light, the resolution of GHOSTEAM is limited by the propagation distance between the emission surface of the STEA and the object (the thickness of the protective 150-nmthick $\mathrm{SiO}_{2}$ layer in this experiment), which is theoretically estimated as submicrometer (see Supplementary Fig. S1).

\section{Polarization-free image}

Polarization impacts the subwavelength imaging (see the dark region where the slits are along the wave polarization in Fig. 2b, c), which is expected due to the electrical field boundary conditions at the subwavelength scale $^{11,13,14}$ (see Supplementary Fig. S2) and hinders accurate resolution of the object's morphology. With advancement of the in-line rotating $\mathrm{THz}$ wave polarization by an external magnetic field, we secured two images with mutually orthogonal polarizations. This feature allows us to achieve a higher-contrast polarization-free image through postfusion of the two images. To do this, 2D Fourier transform was applied to Fig. 2b, c individually to quantify the distribution in the spatial frequency domain $\left|F\left(u_{x}, u_{y}\right)\right|$ along the $x$ and $y$ directions. The amplitude $\left|F\left(u_{x} u_{y}\right)\right|$ as functions of spatial frequencies $u_{x}$ and $u_{y}$ is shown in Fig. 3a, b, which indicates loss of the high spatial frequency $(>10 / \lambda)$ components parallel to $\boldsymbol{E}_{\mathrm{THz}}$ in the polarization images. To acquire polarizationfree $\mathrm{THz}$ image $\mathbf{X}_{\mathrm{F}}$, the $\mathrm{THz}$ ghost images with horizontal polarization $\mathbf{X}_{\mathrm{H}}$ (Fig. 2b) and vertical polarization $\mathbf{X}_{\mathrm{V}}$ (Fig. 2c) were fused by the weighted average method, namely, $\mathbf{X}_{\mathrm{F}}=r \mathbf{X}_{\mathrm{H}}+(1-r) \mathbf{X}_{\mathrm{V}}$, where $r$ represents the weight fraction and lies within $[0,1]$. The total variation (TV), which is a common assessment parameter for image clearness, was chosen to guide the optimization of the fusion process. The TV of $\mathbf{X}_{\mathrm{F}}$ was calculated by

$$
\mathrm{TV}=\left(\sum_{i=2}^{64} \sum_{j=2}^{64}\left[\left(\nabla_{\mathrm{H}} \mathbf{X}_{\mathrm{F}}(i, j)\right)^{2}+\left(\nabla_{\mathrm{V}} \mathbf{X}_{\mathrm{F}}(i, j)\right)^{2}\right]\right)^{1 / 2}
$$

where $\nabla_{\mathrm{H}}$ and $\nabla_{\mathrm{V}}$ are the discretized gradient operators along the horizontal and vertical directions, respectively. At the ratio of $r=0.53$ in Fig. 3c, the minimal TV was achieved, and the corresponding optimal fused image is shown in Fig. 3d. In addition, the SNR (see Supplementary Fig. S13) and directional spatial gradients ${ }^{29}$ of the fused image are also given in Fig. 3c. The directional spatial 


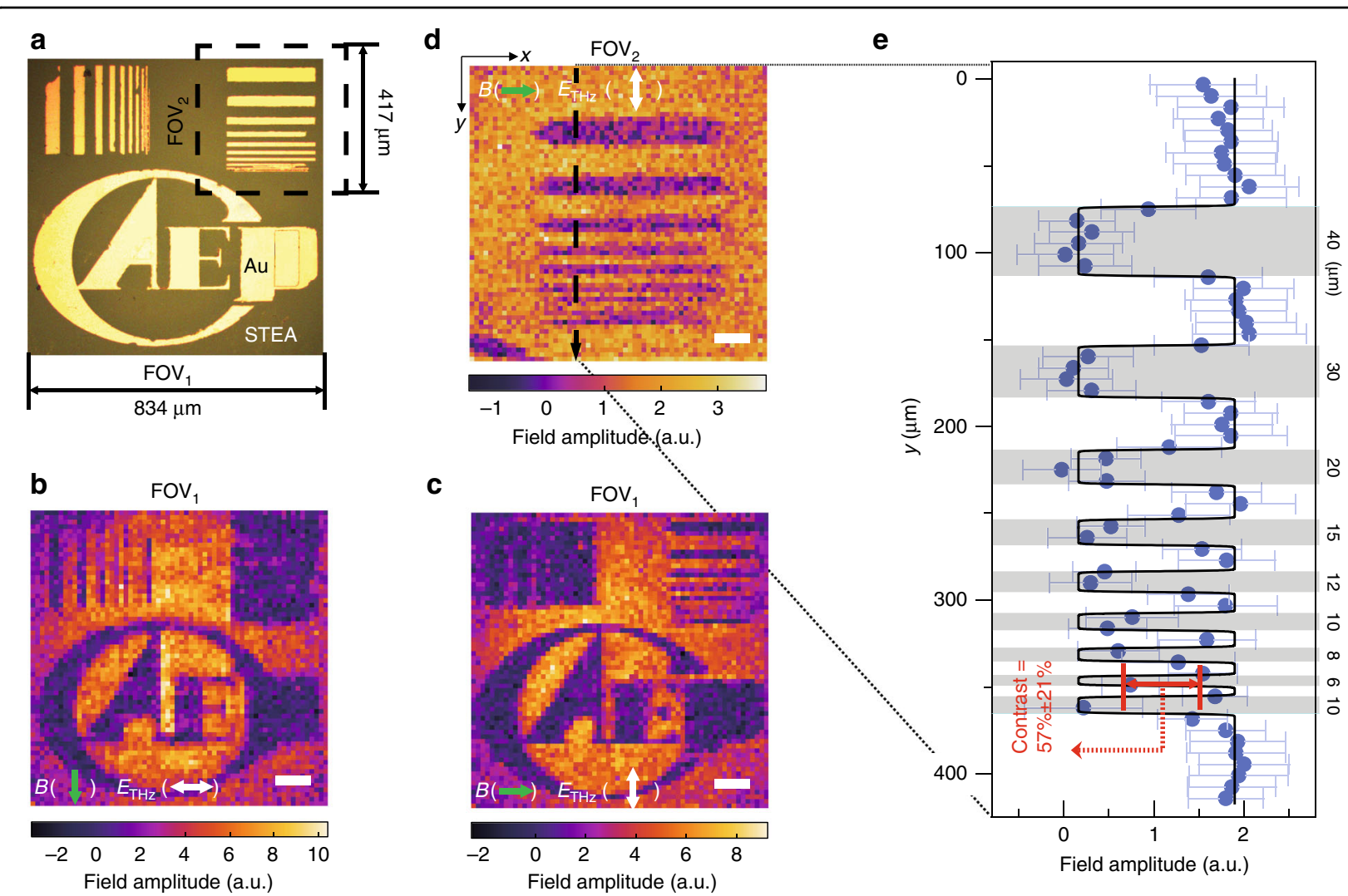

Fig. 2 Subdiffraction-limited images from GHOSTEAM. a Optical image of an object with a field of view of FOV $V_{1}=834 \mu \mathrm{m} \times 834 \mu \mathrm{m}$. The bright regions are gold attached on the 150-nm-thick protective $\mathrm{SiO}_{2}$ layer on top of the STEA. THz ghost images in FOV 1 with a magnetic field (green arrows) applied along the vertical direction (b) and horizontal direction (c). The pixel size and scale bar are $13.0 \mu \mathrm{m}$ and $100 \mu \mathrm{m}$, respectively, for both images. d THz ghost image in $\mathrm{FOV}_{2}$ (indicated by the black dashed box in a) with a pixel size of $6.5 \mu \mathrm{m}$ and a scale bar of $50 \mu \mathrm{m}$. The applied magnetic field $\boldsymbol{B}$ is along the horizontal direction (indicated by the green arrow), and the polarization of the THz radiation (indicated by the white double-headed arrow) is perpendicular to $\boldsymbol{B}$. e Averaged amplitude of the $\mathrm{THz}$ field along the black dashed arrow in (b). Blue dots are averaged experimental data, and the black solid curve is the fit to the Boltzmann sigmoidal function (see Supplementary section 5 for details). Grey areas represent the metal regions, with corresponding widths indicated. A contrast ratio of $57 \pm 21 \%$ is observed at the 6 - $\mu$ m width metal slit.

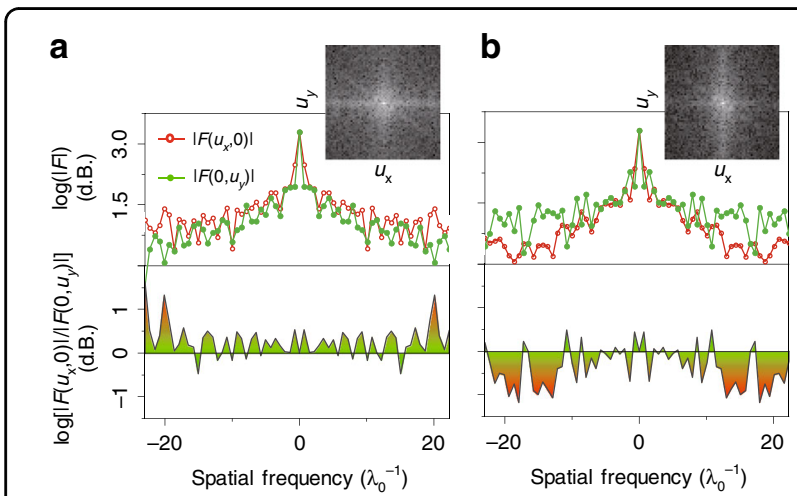

C

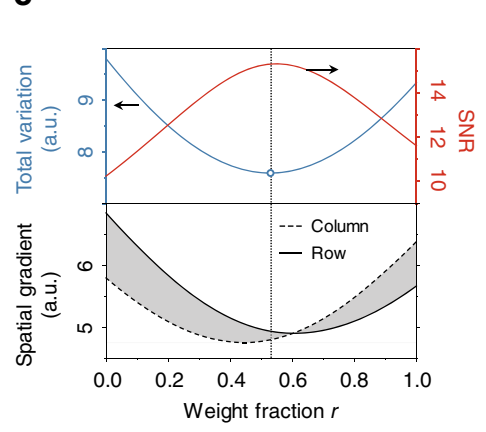

d

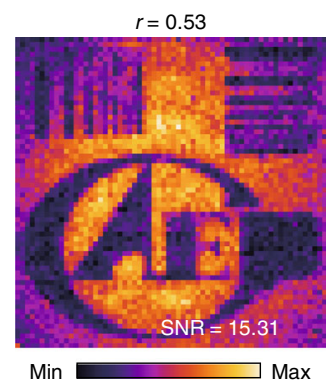

Norm. field amplitude (a.u.)

Fig. 3 Polarization-free THz image. 2D Fourier transform of Fig. $2 b(\mathbf{a})$ and $2 c(\mathbf{b})$. The amplitude $\left|F\left(u_{x}, u_{y}\right)\right|\left(\left|F\left(u_{x \prime} 0\right)\right|\right.$ and $\left|F\left(0, u_{y}\right)\right|$ are in red circles and blue dots, respectively) is shown as a function of the spatial frequency (upper panels). $\log \left[\left|F\left(u_{x}, 0\right)\right| /\left|F\left(0, u_{y}\right)\right|\right]$ is shown in the lower panels for comparison of the horizontal and vertical spatial distributions. c Assessments (total variation and spatial gradient; see Supplementary section 7 for details) of the fused image as a function of weight fraction $r$. The blue open circle indicates the minimum TV $=7.59$ obtained at $r=0.53$. $\mathbf{d}$ Fused polarization-free $\mathrm{THz}$ image with optimized $\mathrm{SNR}=15.31$ at $r=0.53$. 
gradients of $\mathbf{X}_{\mathrm{F}}$ were used to quantify the spatial information of the fused image and were calculated by

$$
\begin{aligned}
& G_{\text {row }}=\left(\sum_{i}^{64} \sum_{j=2}^{64}\left[\left(\nabla_{\mathrm{H}} \mathbf{X}_{\mathrm{F}}(i, j)\right)^{2}\right]\right)^{1 / 2} \\
& G_{\text {col }}=\left(\sum_{i=2}^{64} \sum_{j}^{64}\left[\left(\nabla_{\mathrm{V}} \mathbf{X}_{\mathrm{F}}(i, j)\right)^{2}\right]\right)^{1 / 2}
\end{aligned}
$$

As shown in Fig. 3c, the fused image at $r=0.53$ shows more homogeneous spatial information in the vertical and horizontal directions, as visually presented in Fig. 3d, which proves that the polarization impacts are effectively overcome and that the original object's morphology is better reproduced.

\section{Time-of-fight topography}

Since the $\mathrm{THz}$ emission from the STEA used in our GHOSTEAM system is coherent, TOF measurements ${ }^{30}$ of $\mathrm{THz}$ pulses from the STEA can be adopted to enable microscopic topography of objects with depth resolution. TOF topography utilizes the optical path differences among different media to acquire the depth ( $z$ axis) distribution of a $3 \mathrm{D}$ object. For the sake of illustration, a 3D object made of silica by fs laser manufacturing (see "Materials and methods") with three $\mathrm{SiO}_{2}$ /air interfaces with air depths of 0, 100 and $200 \mu \mathrm{m}$ (illustrated in Fig. 4a) was used to demonstrate TOF microscopic topography using the GHOSTEAM system. The structured silica was attached to the external surface of the STEA. Transient $\mathrm{THz}$ waveforms from the STEA that passed through the object within and outside the region of the trilayer structure were measured and are shown in Fig. $4 \mathrm{~b}$. Three $\mathrm{THz}$ ghost images relevant to the three interfaces, under the field of view $\mathrm{FOV}_{1}$, were measured at fixed time delays of $0,-0.33$ and $-0.66 \mathrm{ps}$ (indicated by the three solid circles in Fig. 4b), as shown in Fig. $4 \mathrm{c}-\mathrm{e}$. The reconstructed images agree with the simulated electrical field distributions (Fig. 4f-h; see "Materials and methods" for simulation details). The subwavelength structures (scale of $\sim \lambda_{0} / 6$ ) of the three interfaces are well resolved both experimentally and theoretically (note that diffraction leads to distortion in reconstruction of the middle interface).

\section{Discussion}

In conclusion, we have presented a novel GHOSTEAM system for $\mathrm{THz}$ wave near-field microscopic topography. Compared with other existing $\mathrm{THz}$ wave near-field imaging systems, the GHOSTEAM system utilizes a realtime, reconfigurable, coherent STEA for structured nearfield illumination. It was numerically and experimentally demonstrated that such a system can feature both micrometre-scale-resolved microscopy $(\leq 6.5 \mu \mathrm{m})$ and depth-resolved topography. Further optimized optical projection imaging systems could enable submicrometrescale resolution. Polarization-free subdiffraction-limited imaging was also achieved owing to the flexible tunability of the STEA.

The capacity of an STE to provide efficient $\mathrm{THz}$ pulses would further enable label-free cellular imaging or ultraprecise topography, a feature lacking in the current $\mathrm{THz}$ near-field imaging systems. STEs fabricated on flexible substrates $^{31}$ would extend our GHOSTEAM system to imaging of objects with curved surfaces. Oscillator $f_{s}$ lasers have been demonstrated to drive STEs with a dynamic range above $60 \mathrm{~dB}(1000: 1)^{26}$. Taking advantage of the $\mathrm{MHz}$ repetition rate and stable pulse energy of oscillators, the GHOSTEAM system is endowed with a much faster acquisition speed (in principle, an 18-fold improvement can be expected; see "Materials and methods" and Supplementary Fig. S9 for the estimation) than all other existing $\mathrm{THz}$ wave subdiffraction ghost imaging systems ${ }^{11-14,16,17}$, which require an amplified fs laser with a few-kilohertz repetition rate, leading to a slow acquisition speed and a complex system. In addition, using a shorter fs pulse and a wide-band detector, the ultrabroadband property of STEs, fully covering $0.1-30 \mathrm{THz}$ frequencies $^{24}$, has great potential for broadband $\mathrm{THz}$ applications. In addition, the GHOSTEAM system is compatible with compressive sensing (see Supplementary GIFs. 1-5), adaptive sampling ${ }^{12,32}$, parallel acquisition in $k$-space ${ }^{33}$ and coherent time-resolved full-wave detection in the time domain ${ }^{16,17}$.

\section{Materials and methods \\ Experimental details}

$\mathrm{THz}$ pulses $(0.2-1.7 \mathrm{THz}$ with a central frequency of $0.5 \mathrm{THz})$ were generated in $\mathrm{W}(2 \mathrm{~nm}) / \mathrm{Fe}(2 \mathrm{~nm}) / \mathrm{Pt}(2 \mathrm{~nm})$ trilayer heterostructures [placed in a rotatable dc field $(|\boldsymbol{B}|=80 \mathrm{mT}$ )] by an $800-\mathrm{nm}$ laser (duration of $90 \mathrm{fs}$, repetition rate of $1 \mathrm{kHz}$, and pump pulse energy of $20 \mu \mathrm{J}$ ). The $\mathrm{THz}$ signals were electrooptically sampled by $1-\mathrm{mm}$ thick ZnTe (110) in combination with a balanced detector and were eventually recorded by a lock-in amplifier with an integration time of $100 \mathrm{~ms}$ (see Supplementary Fig. S4 for details). All the $\mathrm{THz}$ ghost images were multiplexed using the Walsh-Hadamard matrix and operated at respective fixed time delays (e.g., the peak time delays for Fig. $2 \mathrm{~b}-\mathrm{d}$ and appropriate time delays for Fig. 4c-e indicated in Fig. 4b). The acquisition time for each mask was $2 \mathrm{~s}$, and the imaging time for each $64 \times 64$ ghost image (Fig. 2b-d) was $\sim 4.5 \mathrm{~h}$.

To optimize the wavefront of fs pulses, three steps were adopted to determine the positions of $\mathrm{DMD}_{1}$ and $\mathrm{DMD}_{2}$ (Wintech DMD4500, which contains $1140 \times 912$ diamond-arrayed micromirrors with a tilt angle of $12 \pm 1^{\circ}$ and a micromirror pitch of $7.6 \mu \mathrm{m}$ ). First, we acquired the 


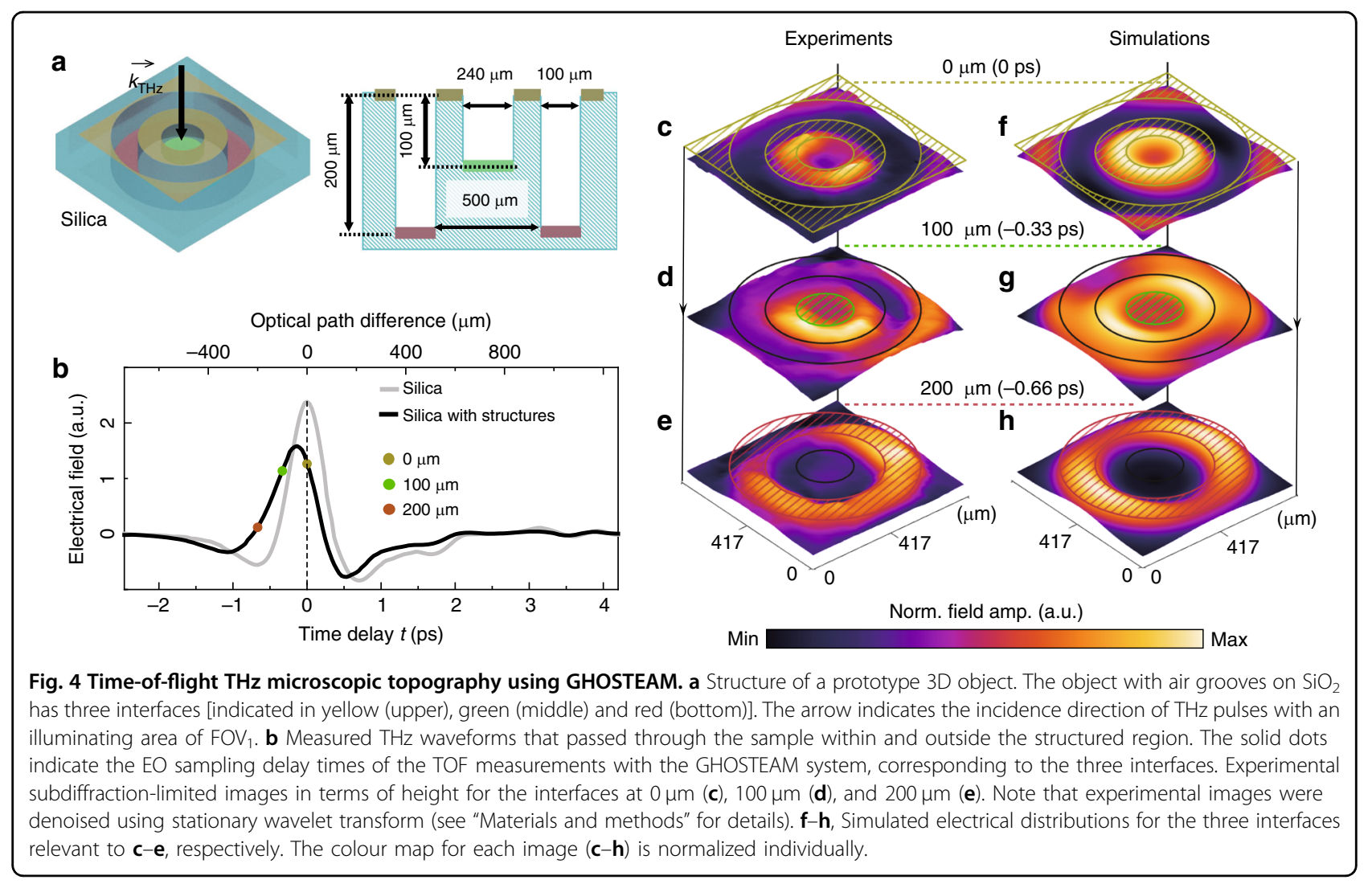

one-to-one image of $\mathrm{DMD}_{2}$ by placing a CCD (see Supplementary Fig. S4c) at the presupposed position of $\mathrm{DMD}_{1}$ to adjust and determine the positions of $\mathrm{DMD}_{2}$ and two lenses (focal lengths of $100 \mathrm{~mm}$ ). Second, by adjusting the position of $\mathrm{DMD}_{1}$, it could be determined when the images (at the presupposed position of the STE) shaped by $\mathrm{DMD}_{1}$ and $\mathrm{DMD}_{2}$ individually were simultaneously sharp. Third, their positions were accurately adjusted by measuring the waveforms of the Walsh-Hadamard masks of \#2p and \#2n (see Supplementary section 3 ) until reaching the minimal peak time delay difference between the two waveforms. The tilt angle difference between $\mathrm{DMD}_{1}$ and $\mathrm{DMD}_{2}$ was measured as $0.40^{\circ}$ by comparing the zero-order diffraction angles of the two DMDs in the case of normal incidence.

\section{Computational ghost imaging}

Let $\mathbf{O}$ represent the pixelated imaging target, consisting of $N$ unknown elements $\mathbf{O}(i)$ at pixel $i$. $\mathbf{O}$ is a vector reshaped from the initial $L \times L$ image matrix $\mathbf{O}_{\mathrm{m}}$, where $L \times L=N$. The DMD is used to display the Walsh-Hadamard masks $\boldsymbol{\phi}_{1}, \boldsymbol{\phi}_{2}, \ldots, \boldsymbol{\phi}_{N}$ in sequence. $\boldsymbol{\phi}_{i}$ with mask number $i(1 \leq i \leq N)$ is an $L \times L$ matrix reshaped from the $i$ th row of the $N$-order Walsh-Hadamard matrix $\Phi$. Then, the correlation between the mask and object, which is recorded by a single-pixel detector, can be mathematically described by their inner product

$$
y_{i}=\left\langle\boldsymbol{\varphi}_{i}, \mathbf{O}>\right.
$$

where $\boldsymbol{\phi}_{i}$ is an $N$-length vector reshaped from $\boldsymbol{\phi}_{i}$. The complete measurement vector is then given by

$$
\mathbf{Y}=\mathbf{\Phi O}
$$

In the experiments, the Walsh-Hadamard matrix consisting of " +1 " and " -1 " elements was realized by

$$
\boldsymbol{\Phi}=\boldsymbol{\Phi}^{(p)}-\boldsymbol{\Phi}^{(n)}
$$

since the DMD can only modulate the amplitude of the incident light. In Equation (6), $\boldsymbol{\Phi}^{(\mathrm{p})}$ is constructed by substituting all " -1 " elements in $\Phi$ with " 0 ". $\Phi^{(\mathrm{n})}$ is acquired by $\Phi^{(\mathrm{p})}-\boldsymbol{\Phi}$.

Ultimately, the ghost image can be reconstructed by

$$
\mathbf{X}=\boldsymbol{\Phi}^{-1} \mathbf{Y}=\boldsymbol{\Phi}^{-1}(\boldsymbol{\Phi} \mathbf{O})=\mathbf{O}
$$

\section{Spatiotemporal THz waveform mapping}

Let $\boldsymbol{E}(\xi, t)$ represent the $\mathrm{THz}$ spatiotemporal waveform to be measured, where $\xi$ and $t$ represent the spatial and 
temporal coordinates, respectively. $\boldsymbol{E}(\mathcal{\xi}, t)$ consists of $N$ time-dependent vectors

$$
\boldsymbol{E}(\xi, t)=\left|\begin{array}{c}
\boldsymbol{E}_{1}(t) \\
\boldsymbol{E}_{2}(t) \\
\vdots \\
\boldsymbol{E}_{N}(t)
\end{array}\right|
$$

The sequentially recorded signals $S(\xi, t)$ can be written as

$$
\boldsymbol{S}(\xi, t)=\left|\begin{array}{c}
\boldsymbol{s}_{1}(t) \\
\boldsymbol{s}_{2}(t) \\
\vdots \\
\boldsymbol{s}_{N}(t)
\end{array}\right|=\boldsymbol{\Phi} \boldsymbol{E}(\xi, t)
$$

Once the complete measurements are obtained, $\boldsymbol{E}(\xi, t)$ can be calculated as

$$
\boldsymbol{E}(\xi, t)=\boldsymbol{\Phi}^{-1} \boldsymbol{S}(\xi, t)
$$

In the experiments, the $i$ th mask had $N=64$ identical rows (columns) for spatiotemporal waveform mapping in the vertical (horizontal) direction, and every row (column) of the mask was the $i$ th row of $\boldsymbol{\Phi}$. The spatial and temporal resolutions were $13 \mu \mathrm{m}$ and $33 \mathrm{fs}$, respectively, for both Fig. 1c, d. The wavefront was then regarded as a linear fit of the peak time delay in $\boldsymbol{E}_{i}(t)$ to the corresponding spatial coordinate $\xi$ (see Supplementary Fig. S5).

\section{Estimation of the SNR and potential frame rate of ghost images}

The SNRs of ghost images obtained via Hadamard multiplexing (Fig. 1f) were estimated by the following equation (see Supplementary section 4 for the detailed mathematical derivation):

$$
\mathrm{SNR}_{\mathrm{H}}=\sqrt{\frac{k}{2 \boldsymbol{N}\left[\gamma_{\mathbf{d}}^{2}+\left(\gamma_{\mathbf{s}} / 2\right)^{2}\right]}}
$$

where $\gamma_{\mathrm{d}}$ denotes the ratio of the dark noise to the $\mathrm{THz}$ peak, $\gamma_{\mathrm{s}}$ denotes the ratio of the peak root mean square error to the THz peak, $N$ denotes the pixel number of the ghost image and $k$ denotes the number of measurements for each mask. In our experimental setup, $\gamma_{\mathrm{d}}$ was measured as $1 \times 10^{-3}$, and $\gamma_{\mathrm{s}}$ was measured as $\sim 7 \times$ $10^{-3}$, as indicated in Fig. 1f. Each mask value was averaged for $k=15$. With these parameters, the $\mathrm{SNR}_{\mathrm{H}}$ was estimated as 11.76 in the case of $N=64 \times 64$, according to Eq. (11).

The frame rate equals FPS $=1 /\left(2 N r_{\mathrm{c}} t_{\text {mask }}\right)$, where $t_{\text {mask }}$ represents the acquisition time for each mask and $r_{\mathrm{c}}$ represents the compressive ratio. $t_{\text {mask }}$ can be expressed as

$$
t_{\text {mask }}=2 N\left(\gamma_{\mathrm{d} 0}^{2}+\gamma_{\mathrm{s} 0}^{2} / 4\right) t_{0} \mathrm{SNR}_{\mathrm{H}}^{2}
$$

where $t_{0}$ denotes the pulse period and $\gamma_{\mathrm{d} 0}$ and $\gamma_{\mathrm{s} 0}$ denote the ratio of the dark noise to a single $\mathrm{THz}$ peak and the pulse fluctuation ratio within the "integration time" of $t_{0}$, respectively. For an $80-\mathrm{MHz}$-oscillator-driven GHOSTEAM system, reasonable values are $t_{0}=12.5 \mathrm{~ns}, \gamma_{\mathrm{d} 0}=$ 2.83 (corresponding to a dark-noise-to-peak ratio of $\gamma_{\mathrm{d}}=$ $\left.1 \times 10^{-3}\right)^{26}$, and $\gamma_{\mathrm{s} 0}=3.8 \times 10^{-3}$ (see Supplementary Fig. S8). With these parameters and $\mathrm{SNR}_{\mathrm{H}}=11.76$, the acquisition time $2 N t_{\text {mask }}$ for a $64 \times 64$ ghost image was calculated as $\sim 15 \mathrm{~min}$, yielding an imaging speed improvement by a factor of $\sim 18(4.5 \mathrm{~h} / 15 \mathrm{~min})$.

\section{Fs laser manufacturing}

The silica sample with three air/silica interfaces for topography was manufactured by the femtosecond laser ablation method. In this method, a Ti-sapphire laser beam (800-nm central wavelength, $30-\mathrm{fs}$ pulse width, $1-\mathrm{kHz}$ repetition rate and $100-\mathrm{mW}$ average power) was focused onto a silica substrate (1-mm thickness) using an objective lens $(10 \times$, numerical aperture $\mathrm{NA}=0.25)$. The silica substrate travelled along multicircular trajectories at a speed of $100 \mu \mathrm{m} / \mathrm{s}$ (original radius $r_{0}$ was $10 \mu \mathrm{m}$, interval between two adjacent circular trajectories $\Delta r$ was $10 \mu \mathrm{m}$, travel number $n_{\mathrm{r}}$ was 10 , and the radius of the inner circular was $\left.r=r_{0}+\Delta r\left(n_{\mathrm{r}}-1\right)=100 \mu \mathrm{m}\right)$. The laser ablation depth under the above parameters was $\sim 50 \mu \mathrm{m}$, and the same ablation process was repeated to obtain an ablation depth of $100 \mu \mathrm{m}$, while the silica substrate was moved $50 \mu \mathrm{m}$ up from the laser spot. Using the same laser fabrication method, the outer circular was produced with $R_{0}=250 \mu \mathrm{m}, \Delta R=10 \mu \mathrm{m}, n_{\mathrm{r}}=15$, and $R=R_{0}+\Delta R\left(n_{\mathrm{r}}-\right.$ $1)=400 \mu \mathrm{m}$. The process was repeated to obtain a deeper interface.

\section{Denoising and simulating near-field ghost topography}

The results of near-field ghost topography (Fig. 4c-e) were denoised using the MATLAB toolbox Stationary Wavelet Transform Denoising 2-D. A five-level Haar wavelet was used to decompose the images. The selected threshold method was penalized low soft thresholding.

The electromagnetic field distributions as the $\mathrm{THz}$ pulse propagates in the near field (Fig. $4 \mathrm{f}-\mathrm{h}$ ) were simulated using the Wave Optics module of the commercial software COMSOL Multiphysics. Certain $\mathrm{THz}$ waves acquired in the experiment were set as the incident source, and the time-dependent solver was used to resolve the electromagnetic field distributions at discrete times (the time step was $33 \mathrm{fs}$ ). The study domain was a rectangle that was divided into silica $\left(n_{\text {silica }}=1.97\right)$ and air $\left(n_{\text {air }}=1\right)$, as illustrated in Fig. 4a. 


\section{Acknowledgements}

This work was supported by the National Key Research and Development Program (No. 2017YFC1200400), the Science Challenge Project (No. TZ2018003), the Distinguished Young Scholars of Sichuan Province (No. 2020JDJQ0008), the National Natural Science Foundation of China (NSFC) (Nos. U1730246 and 11704358), and the Foundation of President of China Academy of Engineering Physics (No. 201501033).

\section{Author details}

${ }^{1}$ Institute of Fluid Physics, China Academy of Engineering Physics, Mianyang 621900 Sichuan, China. 'Department of Optics and Optical Engineering, University of Science and Technology of China, Hefei 230026 Anhui, China. ${ }^{3}$ Microsystem \& Terahertz Research Center, China Academy of Engineering Physics, Chengdu 610200 Sichuan, China. ${ }^{4}$ Beijing National Laboratory for Condensed Matter Physics, Institute of Physics, Chinese Academy of Sciences, 100190 Beijing, China. ${ }^{5}$ National Laboratory of Solid-State Microstructures and Department of Physics, Nanjing University, Nanjing 210093 Jiangsu, China. ${ }^{6}$ Department of Electrical and Computer Engineering, National University of Singapore, 4 Engineering Drive 3, Singapore 117583, Singapore. ${ }^{7}$ The Institute of Optics, University of Rochester, Rochester, NY 14627, USA

\section{Author contributions}

L.-G.Z., S.-C.C., Z.F., J.L. and W.T. conceived the project. S.-C.C., J.L. and Z.-H.Z. designed and performed the imaging experiments. Z.F. and W.T. designed and realized the STE-related module. J.W.C., Y.C.M., K.H. and H.F.D. fabricated the STE samples, 3D silica structure and metal patterns. Z.-R.L., C.-W.Q. and X.-C.Z. helped design the concept. All authors discussed the results and reviewed the manuscript.

\section{Conflict of interest}

L.-G.Z is a named inventor on Chinese patent number 201310442738.9 (publication date 27.05.2015, filing date 26.09.2013). L.-G.Z, J.L, L.-H.D, Z.-H.Z and Z.-R.L are named inventors on Chinese patent number 201410815227.1 (publication date 25.03.2015, filing date 24.12.2014), which is related to the $\mathrm{THz}$ wave ghost microscope. L.-G.Z, S.-C.C, J.L, L.-H.D, Z.-H.Z and Z.-R.L filed a Chinese patent (number 201911281187.6, filing date 11.12.2019), which is related to the concept demonstrated in this work.

Supplementary information is available for this paper at https://doi.org/ 10.1038/s41377-020-0338-4.

Received: 10 March 2020 Revised: 18 May 2020 Accepted: 25 May 2020 Published online: 08 June 2020

\section{References}

1. Ferguson, B. \& Zhang, X. C. Materials for terahertz science and technology. Nat. Mater. 1, 26-33 (2002).

2. Tonouchi, M. Cutting-edge terahertz technology. Nat. Photonics 1, 97-105 (2007).

3. Karpowicz, N. et al. Compact continuous-wave subterahertz system for inspection applications. Appl. Phys. Lett. 86, 054105 (2005).

4. Yang, X. et al. Biomedical applications of terahertz spectroscopy and imaging. Trends Biotechnol. 34, 810-824 (2016).

5. Zou, Y. et al. Label-free monitoring of cell death induced by oxidative stress in living human cells using terahertz ATR spectroscopy. Biomed. Opt. Express $\mathbf{9}$, 14-24 (2018).
6. Zou, Y. et al. Terahertz spectroscopic diagnosis of myelin deficit brain in mice and rhesus monkey with chemometric techniques. Sci. Rep. 7, 5176 (2017).

7. Meng, K. et al. Terahertz pulsed spectroscopy of paraffin-embedded brain glioma. J. Biomed. Opt. 19, 077001 (2014).

8. Huber, A. J. et al. Terahertz near-field nanoscopy of mobile carriers in single semiconductor nanodevices. Nano Lett. 8, 3766-3770 (2008).

9. Cocker, T. L. et al. An ultrafast terahertz scanning tunnelling microscope. Nat. Photonics 7, 620-625 (2013).

10. Kawano, Y. \& Ishibashi, K. An on-chip near-field terahertz probe and detector Nat. Photonics 2, 618-621 (2008).

11. Stantchev, R. I. et al. Noninvasive, near-field terahertz imaging of hidden objects using a single-pixel detector. Sci. Adv. 2, e1600190 (2016).

12. Stantchev, R. I. et al. Compressed sensing with near-field THz radiation. Optica 4, 989-992 (2017).

13. Chen, S. C. et al. Terahertz wave near-field compressive imaging with a spatial resolution of over N100. Opt. Lett. 44, 21-24 (2019).

14. Zhao, J. P. et al. Spatial sampling of terahertz fields with subwavelength accuracy via probe-beam encoding. Light. Sci. Appl. 8, 55 (2019).

15. Watts, C. M. et al. Terahertz compressive imaging with metamaterial spatial light modulators. Nat. Photonics 8, 605-609 (2014).

16. Olivieri, L. et al. Time-resolved nonlinear ghost imaging. ACS Photonics $\mathbf{5}$ 3379-3388 (2018).

17. Olivieri, L. et al. Hyperspectral terahertz microscopy via nonlinear ghost imaging. Optica 7, 186-191 (2020).

18. Erkmen, B. I. \& Shapiro, J. H. Ghost imaging: from quantum to classical to computational. Adv. Opt. Photonics 2, 405-450 (2010).

19. Edgar, M. P., Gibson, G. M. \& Padgett, M. J. Principles and prospects for singlepixel imaging. Nat. Photonics 13, 13-20 (2019).

20. Bethe, H. A. Theory of diffraction by small holes. Phys. Rev. 66, 163-182 (1944).

21. Valenzuela, S. O.\& Tinkham, M. Direct electronic measurement of the spin Hall effect. Nature 442, 176-179 (2006).

22. Sinova, J. et al. Spin Hall effects. Rev. Mod. Phys. 87, 1213-1260 (2015).

23. Kampfrath, T. et al. Terahertz spin current pulses controlled by magnetic heterostructures. Nat. Nanotechnol. 8, 256-260 (2013)

24. Seifert, T. et al. Efficient metallic spintronic emitters of ultrabroadband terahertz radiation. Nat. Photonics 10, 483-488 (2016).

25. Feng, Z. et al. Highly efficient spintronic terahertz emitter enabled by metaldielectric photonic crystal. Adv. Optical Mater. 6, 1800965 (2018).

26. Torosyan, G. et al. Optimized spintronic terahertz emitters based on epitaxial grown Fe/Pt layer structures. Sci. Rep. 8, 1311 (2018).

27. Harwit, M. \& Sloane, N. J. A. Hadamard Transform Optics. (Academic Press, New York, 1979).

28. Murate, K. et al. Adaptive spatiotemporal optical pulse front tilt using a digital micromirror device and its terahertz application. Opt. Lett. 43, 2090-2093 (2018).

29. Eskicioglu, A. M. \& Fisher, P. S. Image quality measures and their performance. IEEE Trans. Commun. 43, 2959-2965 (1995).

30. Zhong, $\mathrm{H}$. et al. Nondestructive defect identification with terahertz time-offlight tomography. IEEE Sens. J. 5, 203-208 (2005).

31. Wu, Y. et al. High-performance $\mathrm{THz}$ emitters based on ferromagnetic/nonmagnetic heterostructures. Adv. Mater. 29, 1603031 (2017).

32. Phillips, D. B. et al. Adaptive foveated single-pixel imaging with dynamic supersampling. Sci. Adv. 3, el601782 (2017).

33. Chun, I. Y. \& Adcock, B. Compressed sensing and parallel acquisition. IEEE Trans. Inf. Theory 63, 4860-4882 (2017). 